\title{
Assessment of Clinical Characteristics and Mortality-Associated Factors in COVID-19 Critical Cases in Kuwait
}

\author{
Mariam Ayed ${ }^{\text {a }}$ Abdulwahab A. Borahmah ${ }^{b}$ Anwar Yazdani ${ }^{c}$ Ahmad Sultan $^{d}$ \\ Ahmad Mossad $^{d}$ Hanouf Rawdhan $^{\mathrm{e}}$ \\ ${ }^{a}$ Neonatal Department, Farwaniya Hospital, Subah-Alnaser, Kuwait; ${ }^{b}$ Department of Anasthesia and Critical Care, \\ Jaber AlAhmad Hospital, Hawali, Kuwait; 'Department of Anaesthesia and Critical Care, Al-Sabah Hospital, \\ Kuwait City, Kuwait; dDepartment of Anaesthesia and Critical Care, Amiri Hospital, Kuwait City, Kuwait; \\ eDepartment of Anesthesia and Critical Care, Infectious Diseases Hospital, Kuwait City, Kuwait
}

\section{Highlights of the Study}

- No significant difference in age and sex was found among critically ill patients with COVID-19.

- This study suggests that the risk factors that can be used to predict mortality include preexisting hypertension, severe acute respiratory distress syndrome, renal replacement therapy, procalcitonin level, serum albumin, lymphocyte count, and D-dimer.

- Early identification of risk factors for mortality might help achieve better outcomes.

\section{Keywords}

SARS-CoV-2 - Coronavirus infection · Pneumonia · Acute respiratory distress syndrome

\begin{abstract}
Objective: The objective of this study was to assess the clinical characteristics and identify mortality risk factors in intensive care unit (ICU)-admitted COVID-19 patients. Methods: We recruited and analyzed SARS-CoV-2-infected adult patients (age $\geq 18$ years) who were admitted to the ICU at Jaber Al-Ahmad Al Sabah Hospital, Kuwait, between March 1, 2020, and April 30, 2020. The risk factors associated with inhospital mortality were assessed using multiple regression
\end{abstract}

karger@karger.com www.karger.com/mpp

Karger $\stackrel{\text { ' }}{5}$

GOPEN ACCESS
(C) 2020 The Author(s)

Published by S. Karger AG, Basel

This is an Open Access article licensed under the Creative Commons Attribution-NonCommercial-4.0 International License (CC BY-NC) (http://www.karger.com/Services/OpenAccessLicense), applicable to the online version of the article only. Usage and distribution for commercial purposes requires written permission. analysis. Results: We recruited a total of 103 ICU patients in this retrospective cohort. The median age of the patients was 53 years and the fatality rate was $45.6 \%$; majority ( $85.5 \%)$ were males and $37 \%$ patients had more than 2 comorbidities. Preexisting hypertension, moderate/severe acute respiratory distress syndrome, lymphocyte count $<0.5 \times 10^{9}$, serum albumin $<22 \mathrm{~g} / \mathrm{L}$, procalcitonin $>0.2 \mathrm{ng} / \mathrm{mL}$, D-dimer $>1,200 \mathrm{ng} / \mathrm{mL}$, and the need for continuous renal replacement therapy were significantly associated with mortality. Conclusion: This study describes the clinical characteristics and risk factors for mortality among ICU patients with $\mathrm{CO}$ VID-19. Early identification of risk factors for mortality might help improve outcomes.

(c) 2020 The Author(s)

Published by S. Karger AG, Basel 


\section{Introduction}

The outbreak of novel coronavirus disease began with a simple case of pneumonia in Wuhan, China, in late December, 2019. By January 30, 2020, the World Health Organization declared the disease as a Public Health Emergency of International Concern and renamed it as COVID-19 [1]. Less than a month later, on February 24, the State of Kuwait confirmed 3 patients on a flight coming from Islamic Republic of Iran to have contracted the virus, marking the start of the outbreak in the country [2]. By March 3, 2020, the first COVID-19 patient was admitted to the intensive care unit (ICU). In the next 2 months, $>100$ confirmed cases of COVID-19 were admitted to the ICU.

The COVID-19 pandemic is considered one of the greatest global public health crises since the 1918 influenza pandemic [3]. Although the clinical spectrum of the infection appears to be broad, patients infected with COVID-19 are found to be at high risk of developing pneumonia, acute respiratory distress syndrome (ARDS), or multi-organ failure, requiring hospitalization and admission in a critical care unit [4-6]. The lack of adequate preparedness and late implementation of the countermeasures led to a drastic global spread of COVID-19 within a short period of time. Furthermore, the lack of an appropriate vaccine has also contributed to the spread and the high mortality of this disease, despite the low mortality rate of the infection itself. Several epidemiological studies have shown that advanced age and presence and history of severe comorbidities, such as cardiovascular diseases, are significantly associated with COVID-19-related mortality. Moreover, COVID-19-related mortality has been attributed to virus-activated "cytokine storm syndrome" [7].

The high rate of spread of this disease has put a lot of burden on an already overwhelmed health-care system. This has led to development of new COVID-19-specific treatment centers and prioritizing the use of medical resources based on patient characteristics. Understanding the risk factors of ICU admission is an important factor in triage decision-making and identification of patients who might benefit more from critical care services [8]. We assessed the clinical characteristics and identified the mortality-associated predictive factors in COVID-19 patients who were admitted to the ICU in Kuwait.

\section{Methods}

\section{Study Design and Settings}

We conducted a retrospective study consisting of patients admitted to the ICU at Jaber Al-Ahmad Hospital, between March 1,
2020, and April 30, 2020. Jaber Al-Ahmad Al Sabah hospital was designated as the national hospital for the management of COVID-19-infected patients in Kuwait. The hospital initially had 3 ICUs (60 beds), and as the pandemic progressed, the ICU bed capacity was subsequently increased to 145 beds.

\section{Patient Criteria}

We enrolled patients aged $\geq 18$ years admitted to ICU with SARS-Cov- 2 infection as confirmed by real-time reverse transcriptase PCR assay of nasopharyngeal swab specimens. Management and treatment of COVID-19 ICU patients were according to the published international and local guidelines [9].

\section{Data Collection and Definition}

Patient data were extracted from the electronic medical records using standardized data collection sheet. We recorded information on age, gender, preexisting medical conditions, including hypertension, diabetes, ischemic heart disease, hyperlipidemia, bronchial asthma, chronic renal failure, malignancy, and autoimmune diseases, home medications, and presenting symptoms prior to admission. We collected laboratory and radiological test results upon admission to ICU. Chest X-ray findings were categorized as normal, unilateral infiltrate, and bilateral infiltrate, and CT-scan findings were categorized as normal, ground glass appearance, and mixed pattern of consolidation. CT scan of the chest and echocardiographs were requested as clinically indicated and at the discretion of the treating physicians. We also recorded data on maximum respiratory support (i.e., low-flow nasal cannula, noninvasive mechanical ventilation, and intubation) and additional adjunctive support, including inhaled nitric oxide, inotropes, renal replacement therapy (RRT), and extracorporeal membrane oxygenation. Information on patient-specific therapies, such as administration of hydroxychloroquine, antivirals, and convalescent plasma, was also collected.

ARDS was defined according to the Berlin guidelines on ARDS [10], and the quick Sequential Failure Assessment (qSOFA) score was calculated upon admission according to the Third International Consensus Definitions Task Force for sepsis and septic shock [11]. Secondary infection was defined as clinical signs of pneumonia or bacteremia and positive culture on blood or body fluid or endotracheal or bronchoalveolar lavage specimens. The study protocol was approved by the Ethics Committee of the Ministry of Health of Kuwait.

\section{Statistical Analysis}

We reported continuous variables as median (interquartile range $[\mathrm{IQR}]$ ) and categorical variables as number and percentage. With mortality as the outcome of interest, differences between deceased patients and discharged patients were analyzed using Fisher's exact test for categorical variables and the Mann-Whitney U test for continuous variables.

We dichotomized the laboratory findings based on the clinical assessment and normal references. We used multivariate logistic regression model to identify risk factors of mortality. Variables that were found to be significant during the univariate analysis were included in the multivariate regression model. Age was included in the final model as it had been described to be a risk factor for mortality in previous studies [7]. Variables with $>50 \%$ missing values were not included in the model (such as high-sensitivity 
[HS] cardiac troponin). The results of the multivariable regression model are expressed as odds ratio (OR) with 95\% confidence interval $(95 \% \mathrm{CI})$. The area under the receiver operating characteristic curve was used to assess the performance of the regression model, and the Hosmer-Lemeshow test was used to test the goodness of fit. Statistical analysis was performed using STATA/IC 14 (STATA Corp., College Station, TX, USA), and 2-tailed $p<0.05$ was considered statistically significant without adjustment for multiple testing.

\section{Results}

\section{Demographic and Clinical Characteristics}

During the study period, a total of 103 patients were admitted. Of those, $56(54.3 \%)$ patients have been discharged and $47(45.6 \%)$ patients died. The median age was 53 years [IQR: $44-63$ ] and majority $85.4 \%(88 / 103)$ of the patients were male. Two or more comorbidities were present in $37 \%(38 / 103)$ of the patients with diabetes mellitus being the most common (39.2\%) followed by hypertension (35\%). The most common presenting symptoms were fever (61\%) followed by cough/chest pain (48\%). The median duration of symptoms was 2 days [IQR: $1-5$ ] and 5 days [IQR: 1-8] prior to hospital and ICU admission, respectively (Table 1 ).

There were no significant differences in age and gender between the discharged and deceased patients. Deceased patients were more likely to have $>2$ comorbidities compared to the discharged patients (53 vs. $21.6 \% ; p=$ 0.005 ). A higher proportion of deceased patients were hypertensive compared to the discharged group (51 vs. $21.4 \% ; p=0.002$ ). Moreover, deceased patients tended to exhibit higher qSOFA score than the discharged patients $(p=0.05)$ (Table 1).

\section{Laboratory and Radiological Findings}

Table 2 shows the laboratory and radiological findings of the patients on admission to ICU. Total lymphocyte counts and serum albumin levels were significantly lower among the deceased patients. Levels of Cr, procalcitonin (PCT), C-reactive protein (CRP), D-dimer, and HS cardiac troponin were significantly elevated in the deceased patients compared to the discharged patients.

The majority of the patients $(84.6 \%)$ exhibited bilateral infiltrates on chest X-ray (Table 2). Only 10 patients had CT scan results of the chest, with 6 of them showing mixed pattern of consolidation and ground glass appearance and 4 showing mainly ground glass appearance. There was no significant difference between the groups on the basis of the radiological findings.

Predictors of Mortality in SARS-Cov-2

Critical Care Patients

\section{Treatment and Clinical Course}

The treatment and clinical course are shown in Table 3. Most patients $(96 \%)$ were treated with antibiotics. Antiviral therapy (ritonavir-lopinavir) was used for $82.5 \%$ of the patients, while oseltamivir was used for $27 \%$ of the patients. Hydroxychloroquine was administered in $78 \%$ of the patients. Among the 103 patients, only $14.6 \%$ received systemic steroids. Majority of the patients (76.7\%) were intubated and mechanically ventilated, and $54 \%$ of the patients were prone positioned. A total of 9 patients $(8.7 \%)$ required extracorporeal membrane oxygenation, 11 patients $(10.7 \%)$ received inhaled nitric oxide, and 3 patients (2.9\%) received convalescent plasma.

Moderate/severe ARDS was present in $76 \%$ of the patients, and $25 \%$ of the patients developed secondary bacterial infection. Inotropic support was used for $71 \%$ of the patients, and the proportion of deceased patients who required inotropic support was significantly higher than that in the discharged patients ( 89.4 vs. $58.5 \% ; p<0.001$ ). RRT was used in $20.4 \%$ of the patients, and 2 of the patients who had preexisting CKD needed RRT. Moreover, among the deceased patients, intubation, RRT, moderate/ severe ARDS, and secondary bacterial infection were observed more frequently than among the discharged patients. Median time to discharge from ICU was 10 days (IQR: 6-15), and median time to death was 13 days (IQR: $7-23)$.

\section{Risk Factors of Mortality}

The multivariate logistic regression analysis showed that the risk factors associated with mortality were preexisting hypertension (OR: 3.2, 95\% CI: 1.2-8.9), moderate/ severe ARDS (OR:3.4, 95\% CI: 1.1-10.8), lymphocyte counts $<0.5 \times 10^{9} / \mathrm{L}$ (OR: 6.1, 95\% CI: 1.2-29.8), albumin $<22$ g/L (OR: 7.5, 95\% CI: 2.1-26.2), PCT >0.2 ng/mL (OR: 3.8, 95\% CI: 1.3-7.8), D-dimer $>1,200 \mathrm{ng} / \mathrm{mL}$ (OR: 5.1, 95\% CI: 1.2-21.6), and the need for RRT (OR: 9.3, 95\% CI: 2.4-36.2) (Table 4). The area under the receiver operating characteristic curve showed a good accuracy in predicting mortality with an AUC equal to 0.82 (95\% CI $0.74-0.90)$ and an overall accuracy of $76.7 \%$. The Hosmer-Lemeshow test showed a good model adequacy $(p=$ $0.56)$.

\section{Discussion}

In this retrospective case series, we described the clinical characteristics and mortality-related risk factors in adult patients with confirmed COVID-19 who were ad- 
Table 1. Baseline demographics and clinical characteristics

\begin{tabular}{|c|c|c|c|c|}
\hline & $\begin{array}{l}\text { All patients } \\
N=103\end{array}$ & $\begin{array}{l}\text { Discharged group } \\
N=56\end{array}$ & $\begin{array}{l}\text { Death group } \\
N=47\end{array}$ & $p$ value \\
\hline Age, median (IQR) & $53[44-63]$ & $51(40-61)$ & $56(48-63)$ & 0.057 \\
\hline$<40$ & $17(16.5)$ & $15(26.8)$ & $2(4.2)$ & \\
\hline $41-50$ & $29(28.2)$ & $14(25)$ & $15(32)$ & \\
\hline $51-60$ & $30(29)$ & $14(25)$ & $15(33)$ & 0.052 \\
\hline$>71$ & $8(7.7)$ & $3(5.3)$ & $5(10.6)$ & \\
\hline \multicolumn{5}{|l|}{ Sex, $n(\%)$} \\
\hline Male & $88(85.5)$ & $46(82.1)$ & $42(89.4)$ & 0.403 \\
\hline Female & $15(14.5)$ & $10(17.7)$ & $5(10.6)$ & \\
\hline \multicolumn{5}{|l|}{ Number of comorbidities, $n(\%)$} \\
\hline None & $37(36.3)$ & $27(49.1)$ & $10(21.3)$ & \\
\hline Hypertension & $36(35)$ & $12(21.4)$ & $24(51.1)$ & 0.002 \\
\hline Ischemic heart disease & $12(11.8)$ & $6(11)$ & $8(17)$ & 0.119 \\
\hline Dyslipidemia & $11(10.8)$ & $6(11)$ & $5(10.5)$ & 1 \\
\hline Asthma & $13(12.7)$ & $4(7.3)$ & $8(17)$ & 0.231 \\
\hline Malignancy & $3(2.9)$ & $2(3.7)$ & $1(2)$ & 1 \\
\hline Chronic renal failure & $4(3.9)$ & 0 & $4(8.5)$ & 0.056 \\
\hline Others (autoimmune, hypothyroidism) & $8(7.8)$ & $5(9.2)$ & $3(6.4)$ & 0.714 \\
\hline \multicolumn{5}{|l|}{ Home medications, $n(\%)$} \\
\hline Insulin & $34(33.3)$ & $15(26.8)$ & $19(41.3)$ & 0.122 \\
\hline Beta blockers & $13(12.7)$ & $4(7.1)$ & $9(19.6)$ & 0.061 \\
\hline ACEI & $10(9.8)$ & $5(8.9)$ & $5(10.8)$ & 0.734 \\
\hline Aspirin & $7(6.8)$ & $2(3.6)$ & $5(10.8)$ & 0.147 \\
\hline Vomiting/diarrhea & $1(1)$ & 0 & 0 & \\
\hline Fatigue/myalgia & $15(15)$ & $6(11)$ & $6(12.7)$ & 1 \\
\hline Travel history prior to admission, $n(\%)$ & $12(11.6)$ & $10(17.8)$ & $2(4.2)$ & 0.068 \\
\hline Contact with COVID-19-positive patient, $n(\%)$ & $16(15.5)$ & $7(12.5)$ & $9(19)$ & 0.584 \\
\hline Duration of symptoms prior to hospital admission, days, median (IQR) & $2(1-5)$ & $1.5(1-5)$ & $2(1-5)$ & 0.876 \\
\hline Duration of symptoms prior to ICU admission, days, median (IQR) & $5(1-8)$ & $5(1-8)$ & $4(1-8)$ & 0.987 \\
\hline \multicolumn{5}{|l|}{ qSOFA points, $n(\%)$} \\
\hline 1 point & $55(53.4)$ & $31(55.3)$ & $22(46.8)$ & \\
\hline 2 points & $24(23.3)$ & $10(17.8)$ & $14(29.8)$ & 0.05 \\
\hline 3 points & $24(23.3)$ & $13(23.2)$ & $11(23.4)$ & \\
\hline$>2$ points & $48(46.6)$ & $23(41)$ & $25(53.2)$ & 0.240 \\
\hline
\end{tabular}

Data expressed as $n(\%)$ and median (IQR). ACEI, angiotensin-converting enzyme inhibitors; qSOFA, quick Sequential Failure Assessment; IQR, interquartile range; ICU, intensive care unit. 
Table 2. Laboratory and radiological findings on admission

\begin{tabular}{|c|c|c|c|c|}
\hline & All & Discharged group & Death group & $p$ value \\
\hline \multicolumn{5}{|l|}{ Laboratory values } \\
\hline WBC $\left(\times 10^{9} / \mathrm{L}\right)$, median $(\mathrm{IQR})$ & $8.2(6.3,10.2)$ & $8.4(5.5-10)$ & $7.5(6.3-10.6)$ & 0.837 \\
\hline Neutrophils $\left(\times 10^{9} / \mathrm{L}\right)$, median (IQR) & $6.4(4.7,8.7)$ & $6.9(4-8.8)$ & $5.7(4.9-10.1)$ & 0.788 \\
\hline Lymphocytes $\left(\times 10^{9} / \mathrm{L}\right)$, median (IQR) & $0.9(0.7,1.2)$ & $1(0.8-1.2)$ & $0.8(0.5-1.1)$ & 0.005 \\
\hline Lymphocytes $<0.5, n(\%)$ & $15(15.3 \%)$ & $3(5.7 \%)$ & $12(26.1 \%)$ & 0.005 \\
\hline Hemoglobin (g/L), median (IQR) & $122(102-135)$ & $125(109-135)$ & $116(91-135)$ & 0.181 \\
\hline Platelets $\left(\times 10^{9} / \mathrm{L}\right)$, median $(\mathrm{IQR})$ & $238(192-303)$ & $258(204-327)$ & $215(167-269)$ & 0.067 \\
\hline PT INR, median (IQR) & $1.04(0.9-1.1)$ & $1.03(0.97-1.1)$ & $1.07(0.99-1.5)$ & 0.164 \\
\hline aPTT, seconds, median (IQR) & $41.5(20.5-56)$ & $40(21-54)$ & $45(17-59)$ & 0.658 \\
\hline D-dimer, ng/mL, $N=56$, median (IQR) & $1,234(566-2,855)$ & $809(555-1,344)$ & $2,346(893-3,940)$ & 0.029 \\
\hline D-dimer $>1,200 \mathrm{ng} / \mathrm{mL}, n(\%)$ & $28(50 \%)$ & $9(32 \%)$ & $19(67.9 \%)$ & 0.015 \\
\hline Fibrinogen, g/L, median (IQR) & $7(6-7.8)$ & $7(6-7)$ & $6.5(3.5-9)$ & 0.913 \\
\hline $\mathrm{Cr}, \mathrm{mmol} / \mathrm{L}$, median $(\mathrm{IQR})$ & $85(68-116)$ & $82(68-97)$ & $97(77-176)$ & 0.01 \\
\hline $\mathrm{Cr}>120 \mathrm{mml} / \mathrm{L}, n(\%)$ & $22(22.4 \%)$ & $8(15 \%)$ & $14(31 \%)$ & 0.03 \\
\hline Albumin, g/L, median (IQR) & $25.4(22-29)$ & $26(23.6-29.8)$ & $24.5(20.6-28.5)$ & 0.007 \\
\hline Albumin $<22 \mathrm{~g} / \mathrm{L}, n(\%)$ & $24(23.3 \%)$ & $7(13.5 \%)$ & $17(40.5 \%)$ & 0.006 \\
\hline ALT, IU/L, median (IQR) & $42(24-57)$ & $39(23-54)$ & $44(23-65)$ & 0.318 \\
\hline AST, IU/L, median (IQR) & $50(38-74)$ & $45(33-69)$ & $49(40-76)$ & 0.184 \\
\hline LDH, IU/L, median (IQR) & $447(346-561)$ & $445.5(332-633)$ & $453(366-547)$ & 0.527 \\
\hline Troponin I (HS), ng/L, $N=28$, median (IQR) & $22(9-84.7)$ & $9(6-17)$ & $73(23-146)$ & 0.009 \\
\hline Troponin I, ng/L, median (IQR) & $0.013(0.01-0.03)$ & $0.03(0.01-0.04)$ & $0.013(0.003-0.05)$ & 0.538 \\
\hline CK, IU/L, median (IQR) & $296(97-604)$ & $384(87-404)$ & $128.5(104-282)$ & 0.668 \\
\hline Ferritin, ng/mL, median (IQR) & $1,164(681-1,571)$ & $1,425(883-1,588)$ & $1,153(454-1,556)$ & 0.171 \\
\hline $\mathrm{PCT}, \mathrm{ng} / \mathrm{mL}$, median (IQR) & $0.41(0.2-1.5)$ & $0.29(0.09-0.52)$ & $0.81(0.32-3.2)$ & 0.0004 \\
\hline $\mathrm{PCT}>0.2 \mathrm{ng} / \mathrm{mL}, n(\%)$ & $52(49.5 \%)$ & $18(33.3 \%)$ & $28(59.6 \%)$ & 0.036 \\
\hline CRP, mg/L, median (IQR) & $139(95-232)$ & $125(95-166)$ & $164(107-340)$ & 0.009 \\
\hline $\mathrm{CRP}>200 \mathrm{mg} / \mathrm{L}, n(\%)$ & $35(34 \%)$ & $8(14.8 \%)$ & $22(46.8 \%)$ & 0.002 \\
\hline \multicolumn{5}{|l|}{ Radiological findings, $n(\%)$} \\
\hline \multicolumn{5}{|l|}{ Chest X-ray } \\
\hline Unilateral infiltrate & $6(5.7)$ & $3(6.4)$ & $3(6.7)$ & 0.725 \\
\hline Bilateral infiltrate & $88(84.6)$ & $41(87.2)$ & $36(80)$ & \\
\hline \multicolumn{5}{|l|}{ Chest CT scan, $N=10$} \\
\hline Ground glass appearance plus consolidation & $6(60)$ & $2(66.7)$ & $3(50)$ & 1 \\
\hline Ground glass appearance & $4(40)$ & $1(33.3)$ & $3(50)$ & \\
\hline
\end{tabular}

Values are presented in median (IQR) and $n(\%)$. WBC, white blood count; PT INR, prothrombin time international normalized ratio; aPTT, activated partial thromboplastin time; ALT, alanine transaminase; AST, aspartate transaminase; LDH, lactate dehydrogenase; CK, Cr kinase; PCT, procalcitonin; CRP, C-reactive protein; IQR, interquartile range.

mitted to the critical care unit. The major outcome assessed here was patient mortality. We evaluated the association between the patient's demographic, laboratory, and clinical data and mortality. The median age of our patients was 53 years and majority were male. The relationship between gender and mortality in sepsis remained controversial. Results from meta-analysis indicated that males constituted a significantly higher proportion of those who had adverse clinical outcomes and died from COVID-19 [12]. In contrast, no correlation between mortality and gender were observed in other studies. However, unlike other studies, we did not find any sig- nificant correlation between advancing age and patient mortality. This finding could be attributed to the fact that most of the patients recruited in the current study were aged between 44 and 63 years, while other studies include higher proportions of individuals $>65$ years of age, which results in a more significant association between age and mortality $[7,13]$.

Several studies have shown that presence of comorbidities, such as cardiovascular disease and secondary infections, contributes significantly to disease severity and mortality among COVID-19 patients $[7,14,15]$. In an earlier study, hypertension was the most common co- 
Table 3. Treatment and clinical course

\begin{tabular}{|c|c|c|c|c|}
\hline & All & Discharged & Death & $p$ value \\
\hline Lopinavir-ritonavir, $n(\%)$ & $82(79.6)$ & 47 (83.9) & $38(80.8)$ & 0.184 \\
\hline Oseltamivir, $n(\%)$ & $28(27.2)$ & $18(32)$ & $10(21.2)$ & 0.251 \\
\hline Hydroxychloroquine, $n(\%)$ & $79(76.7)$ & $41(73.2)$ & $40(85)$ & 0.151 \\
\hline Antibiotics, $n(\%)$ & $99(96)$ & $45(96)$ & $45(100)$ & 0.495 \\
\hline Inotropic support, $n(\%)$ & $73(70.9)$ & $31(55.3)$ & $42(89.4)$ & 0.001 \\
\hline Systemic steroid, $n(\%)$ & $15(14.6)$ & 7 (12.9) & $8(17)$ & 0.782 \\
\hline Convalescent plasma transfusion, $n(\%)$ & $3(3)$ & 0 & $3(2)$ & 0.092 \\
\hline \multicolumn{5}{|l|}{ Maximum respiratory support, $n(\%)$} \\
\hline High-flow nasal cannula & $22(21.4)$ & $17(30.3)$ & 0 & \\
\hline Noninvasive ventilation & $2(1.9)$ & $2(4.2)$ & 0 & \\
\hline Intubation & $79(76.7)$ & $35(62.5)$ & $47(100)$ & 0.001 \\
\hline Prone positioning, $N=95, n(\%)$ & $51(53.7)$ & $27(51.9)$ & $27(60)$ & 0.518 \\
\hline $\mathrm{ECMO}, n(\%)$ & $9(8.7)$ & $7(12.5)$ & $2(4.3)$ & 0.176 \\
\hline $\mathrm{iNO}, n(\%)$ & $11(0.7)$ & $6(10.7)$ & $5(10.6)$ & 0.737 \\
\hline RRT, $n(\%)$ & $28(27.2)$ & $9(16.1)$ & $19(40.4)$ & 0.006 \\
\hline \multicolumn{5}{|l|}{ ARDS, $N=96, n(\%)$} \\
\hline None & $11(11.5)$ & $11(21.6)$ & 0 & \\
\hline Mild & $12(12.5)$ & $8(15.7)$ & $4(8.9)$ & 0.002 \\
\hline Moderate & $53(55)$ & $24(47)$ & $29(64.4)$ & \\
\hline Severe & $20(21)$ & $8(15.7)$ & $12(26.7)$ & \\
\hline Moderate-severe ARDS, $n(\%)$ & $73(76)$ & $32(62.7)$ & $41(91.1)$ & 0.001 \\
\hline Secondary bacterial infection, $n(\%)$ & $26(25.2)$ & $7(12.5)$ & $16(34)$ & 0.03 \\
\hline Length of ICU stay, days, median (IQR) & $11(6-18.5)$ & $10(6-15)$ & $13(7-23)$ & 0.244 \\
\hline
\end{tabular}

Data are presented in $n(\%)$ and median (IQR). ARDS, acute respiratory distress syndrome; ECMO, extracorporeal membrane oxygenation; iNO, inhaled nitric oxide; RRT, renal replacement therapy; ARDS, acute respiratory distress syndrome; IQR, interquartile range; ICU, intensive care unit.

Table 4. Multivariate analysis for the risk factors of mortality

\begin{tabular}{ll}
\hline Risk factor & OR $(95 \% \mathrm{CI}) ; p$ value \\
\hline Age & \\
$\quad$ Less than 40 years & Reference \\
$\quad 41-50$ & $5.5(0.95-31.9) ; 0.057$ \\
$51-60$ & $5.4(9.95-31.2) ; 0.058$ \\
$\quad 61-70$ & $3.5(0.55-21.8) ; 0.187$ \\
$\quad$ More than 71 years & $4.7(0.51-44.2) ; 0.174$ \\
More than 2 comorbidities & $2.9(0.9-9.2) ; 0.072$ \\
Preexisting hypertension & $3.2(1.2-8.9) ; 0.02$ \\
qSOFA $>2$ points & $2.2(0.95-5.2) ; 0.065$ \\
Moderate to severe ARDS & $3.4(1.1-10.8) ; 0.038$ \\
Cr $>120$ mmol/L & $3.1(0.92-9.9) ; 0.069$ \\
Albumin $<22$ g/L & $7.5(2.1-26.2) ; 0.002$ \\
Lymphocyte counts $<0.5 \times 10^{9}$ & $6.1(1.2-29.8) ; 0.026$ \\
PCT $>0.2$ ng/mL & $3.8(1.3-7.8) ; 0.014$ \\
CRP $>200$ ng/mL & $2.3(0.78-6.8) ; 0.133$ \\
D-dimer $>1,200$ ng/mL & $5.1(1.2-21.6) ; 0.026$ \\
Sepsis & $2.7(0.96-7.9) ; 0.057$ \\
Intubation & $2.1(0.95-4.6) ; 0.064$ \\
Inotropic support & $3.5(0.87-14.1) ; 0.078$ \\
RRT & $9.3(2.4-36.2) ; 0.001$ \\
\hline
\end{tabular}

morbidity, affecting 509 (49\% [95\% CI, 46-52\%]) of 1,043 patients with available data [16]. We found that $37 \%$ of the patients who were admitted to the ICU exhibited presence of previous comorbidities, the most common being diabetes (39.2\%) and hypertension (35\%). Similarly, half of the patients who died suffered from hypertension (51.1\%) and diabetes (48.9\%). This finding was consistent with those of the previous studies [15]. Furthermore, our results also indicated hypertension to be an independent risk factor for mortality (adjusted OR: 3.2, 95\% CI: 1.2-8.9). Moreover, compared to the discharge group, more than double the number of patients in the death group suffered from 2 or more comorbidities.

In several studies, ICU mortality has been associated with organ dysfunction. qSOFA is a recently developed scoring system used to assess the organ dysfunction. Although qSOFA score does not directly evaluate patient mortality, previous studies have demonstrated the relationship between mortality and dysfunction [17]. In our study as well, a higher proportion of the patients who died showed higher qSOFA scores compared to the patients in 
the discharged group (53.2 vs. $41.1 \%$, respectively); however, the difference was not statistically significant $(p=$ $0.24)$.

Our results also indicated that patient mortality was significantly associated with higher levels of HS troponin, PCT, and CRP. Elevated levels of HS troponin have been associated with acute coronary syndrome, reduced left ventricular function, and higher levels of IL- 6 and TNF-alpha, which ultimately leads to mortality among the ICU patients [18]. PCT, a precursor of calcitonin, is mainly released during bacterial sepsis and tissue injury. It has previously been recognized as an indicator of severity of organ failure and mortality among the ICU patients $[19,20]$. It is noteworthy that high levels of PCT are associated with bacterial infection, which indicates that the death among the COVID-19 patients with elevated PCT might be due to secondary bacterial infections. This assumption was supported by our results which showed that compared to the discharge group, a higher proportion of patients who died suffered from bacterial infections. CRP is involved in inflammatory reactions, and elevated CRP levels have been significantly associated with several cardiovascular disorders, organ failure, and mortality among the ICU patients $[21,22]$.

Lower levels of lymphocytes (lymphopenia) and albumin (hypoalbuminemia) have also been associated with patient mortality. Within the death group, 40.5 and $13.5 \%$ of the patients exhibited albumin $<22 \mathrm{~g} / \mathrm{L}$ and lymphocytes $<0.5 \times 10^{9} / \mathrm{L}$, which was significantly higher compared to the discharge group (26.1 and 5.7\%, respectively) $(p<0.05)$. Our results corroborated with those of the previous studies [23, 24]. Furthermore, D-dimer has been previously shown to be a sensitive independent predictor of mortality among ICU patients [25], which was consistent with our results. Compared to the discharged patients, a significantly higher proportion of patients who died had D-dimer $>1,200 \mathrm{ng} / \mathrm{mL}(p=0.015)$. However, a few studies have discouraged the use of $\mathrm{D}$-dimer quantification for mortality prediction in ICU patients $[25,26]$. In addition, requirement of RRT was also found to be significantly associated with patient mortality, even after adjusting for the confounding factors (adjusted OR: 9.3, 95\% CI: 2.4-36.2). It is worth mentioning that although high-flow nasal cannula and noninvasive mechanical ventilation were used in only 21 and $2 \%$ of our patients, respectively, none of them died, which could indicate a survival benefit for patients who receive noninvasive ventilation; however, it is very difficult to draw this conclusion at this point due to the nature of our study, and such

Predictors of Mortality in SARS-Cov-2

Critical Care Patients results could be confounded. To our knowledge, this is the first study in our region describing the characteristics of critically ill COVID-19 patients and their predictors for mortality.

\section{Limitations}

There were several limitations for our study. Firstly, the sample size in our study was relatively small. Recruitment of a higher number of patients could have provided a clearer picture. Secondly, the patient data were collected retrospectively from their electronic records, which were often incomplete. This could lead to information bias. Thirdly, there was no standard protocol for laboratory workup of the patients during ICU admission. This led to the absence of the results for some laboratory tests for some patients. Due to this, the laboratory-based data for some patients could not be included in the regression model, which might have led to discrepancy with respect to mortality predictors. In future studies, we propose to recruit larger numbers of patients and to follow them up for a longer duration to further elucidate the factors that contribute to mortality among COVID-19 patients.

\section{Conclusions}

Our observational study involving critically ill patients with confirmed COVID-19 showed no significant differences in mortality based on age and gender. Several risk factors were identified to predict mortality. With the increasing surge of critical care patients, early identification of risk factors for mortality is crucial in triage decisionmaking and for proper utilization of resources. More multicentric large studies are needed to validate the significance of these predictors with respect to patient mortality.

\section{Acknowledgements}

We thank Dr. Abdulrahman Alrefai, Dr. Ali Bander, and Dr. Abdulrahman Alfares who designed the record sheet for data collection.

\section{Statement of Ethics}

The study was approved by the Ethics Committee of the Ministry of Health of Kuwait (2020/1411) 


\section{Conflict of Interest Statement}

The authors declare to have no conflicts of interests.

\section{Funding Sources}

This study did not receive any specific grants.

\section{Author Contributions}

Study design: Mariam Ayed, Abdulwahab A. Borahmah, Anwar Yazdani, Ahmad Sultan, Ahmad Mossad, and Hanouf Rawdhan; data collection: Mariam Ayed, Abdulwahab A. Borahmah, Anwar Yazdani, Ahmad Sultan, Ahmad Mossad, and Hanouf Rawdhan; data analysis: Mariam Ayed; drafting of manuscript: Mariam Ayed; revision of manuscript: Mariam Ayed, Abdulwahab A. Borahmah, Anwar Yazdani, Ahmad Sultan, Ahmad Mossad, and Hanouf Rawdhan; consent for final manuscript: Mariam Ayed, Abdulwahab A. Borahmah, Anwar Yazdani, Ahmad Sultan, Ahmad Mossad, and Hanouf Rawdhan.

\section{References}

1 Outbreak was declared a Public Health Emergency of International concern by WHO. 2020 Jan 30. Available from: https://www. who.int/emergencies/diseases/novel-coronavirus-2019/events-as-they-happen.

2 Kuwait confirms 3 coronavirus patients coming from Iran. $2020 \mathrm{Feb} 24$. Available from: https: //www.kuna.net.kw/ArticleDetails. aspx $? \mathrm{id}=2864385$ \&language $=$ en .

3 Sanders JM, Monogue ML, Jodlowski TZ, Cutrell JB. Pharmacologic treatments for coronavirus Disease 2019 (COVID-19): a review. JAMA. 2020;323(18):1824-36.

4 Yang X, Yu Y, Xu J, Shu H, Xia J, Liu H, et al. Clinical course and outcomes of critically ill patients with SARS-CoV-2 pneumonia in Wuhan, China: a single-centered, retrospective, observational study. Lancet Respir Med. 2020;8(5):475-81.

5 Zhong NS, Zheng BJ, Li YM, Poon LLM, Xie $\mathrm{ZH}$, Chen $\mathrm{KH}$, et al. Epidemiology and cause of severe acute respiratory syndrome (SARS) in Guangdong, People's Republic of China, in February, 2003. Lancet. 2003;362(9393): 1353-8.

6 Wang N, Shi X, Jiang L, Zhang S, Wang D, Tong P, et al. Structure of MERS-CoV spike receptor-binding domain complexed with human receptor DPP4. Cell Res. 2013;23(8): 986-93.

7 Ruan Q, Yang K, Wang W, Jing L, Song J. Clinical predictors of mortality due to COVID-19 based on an analysis of data of $150 \mathrm{pa}-$ tients from Wuhan, China. Intensive Care Med. 2020;46(5):846-8.

8 Bhatraju PK, Ghassemieh BJ, Nichols M, Kim $\mathrm{R}$, Jerome KR, Nalla AK, et al. Covid-19 in critically ill patients in the Seattle region: case series. N Engl Med. 2020;382(21):2012-22.

9 Bartlett RH, Ogino MT, Brodie D, McMullan $\mathrm{DM}$, Lorusso R, MacLaren $\mathrm{G}$, et al. Initial ELSO guidance document: ECMO for COV-
ID-19 patients with severe cardiopulmonary failure. JAMA. 2020;66(5):472-4.

10 Ranieri VM, Rubenfeld GD, Thompson BT, Ferguson ND, Cladwell E, Fan E, et al. Acute respiratory distress syndrome: the Berlin definitions. JAMA. 2012;307(23):2526-33.

11 Singer M, Deutschman CS, Seymour CW, Shankar-Hari M, Annane D, Bauer M, et al. The third international consensus definitions for sepsis and septic shock (Sepsis-3). JAMA. 2016;315(8):801-10.

12 Galbadage T, Peterson BM, Awada J, Buck AS, Ramirez DA, Wilson J, et al. Systematic review and meta-analysis of sex-specific COVID-19 clinical outcomes. Front Med. 2020; $7: 348$.

13 Wang W, Tang J, Wei F. Updated understanding of the outbreak of 2019 novel coronavirus (2019-nCoV) in Wuhan, China. J Med Virol. 2020;92(4):441-7.

14 Li B, Yang J, Zhao F, Zhi L, Wang X, Liu L, et al. Prevalence and impact of cardiovascular metabolic diseaseson COVID-19 in China. Clin Res Cardiol. 2020;109:531-8.

15 Yang J, Zheng Y, Gou X, Pu K, Chen Z, Guo $\mathrm{Q}$, et al. Prevalence of comorbidities and its effects in patients infected with SARS-CoV-2: a systematic review and meta-analysis. Int $\mathrm{J}$ Infect Dis. 2020;94:91-5.

16 Grasselli G, Zangrillo A, Zanella A, Antonelli $\mathrm{M}$, Cabrini L, Castelli A, et al. Baseline characteristics and outcomes of 1591 patients infected with SARS-CoV-2 admitted to ICUs of the Lombardy region, Italy. JAMA. 2020; 323(16):1574-81.

17 Ferreira FL, Bota DP, Bross A, Melot C, Vincet J-L. Serial evaluation of the SOFA score to predict outcome in critically ill patients. JAMA. 2001;286(14):1754-8.

18 Ammann P, Maggiorini M, Bertel O, Haenseler E, Jemelka HI, Oechslin E, et al. Troponin as a risk factor for mortality in critically ill pa- tients without acute coronary syndromes. J Am Coll Cardiol. 2003;41(11):2004-9.

19 Hatherill M, Tibby SM, Turner C, Ratnavel N, Murdoch IA. Procalcitonin and cytokine levels: relationship to organ failure and mortality in pediatric septic shock. Crit Care Med. 2000;28(7):2591-4.

20 Jensen JU, Heslet L, Jensen TH, Espersen K, SteffensenTavede PM. Procalcitonin increase in early identification of critically ill patients at high risk of mortality. Crit Care Med. 2006; 34(10):2596-602.

21 The Emerging Risk Factors Collaboration. Creactive protein concentration and risk of coronary heart disease, stroke, and mortality: an individual participant meta-analysis. Lancet. 2010;375(9709):132-40.

22 Lobo S, Lobo F, Bota DP, Ferreira F, Melot C, Vincent J-L. C-reactive protein levels correlate with mortality and organ failure in critically ill patients. Chest. 2003;123(6):2043-9.

23 Yap FHY, Joynt GM, Buckley TA, Wong ELY. Association of serum albumin concentration and mortality risk in critically ill patients. Anaesth Intensive Care. 2002;30(2):202-7.

24 Jiménez-Ibáñez EO, Castillejos-Lopez M, Hernandez A, Gorocica P, Alvarado-Vasquez N. High mortality associated with hyperglycemia, neutrophilia, and lymphopenia in critically ill patients. Tohoku J Exp Med. 2012; 226(3):213-20.

25 Shorr AF, Trotta RF, Alkins SA, Hanzel GS, Diehl LF. D-dimer assay predicts mortality in critically ill patients without disseminated intravascular coagulation or venous thromboembolic disease. Intensive Care Med. 1999; 25(2):207-10.

26 Pettilä V, Hynninen M, Takkunen O, Kuusela $\mathrm{P}$, Valtonen M. Predictive value of procalcitonin and interleukin 6 in critically ill patients with suspected sepsis. Intensive Care Med. 2002;28(9):1220-5 